\section{Preparation of 2" X 2" Projection Slides From EM And Other Negatives}

A. Kent Christensen

University of Michigan Medical School

In the early days of biological electron microscopy (the 1950 s and 1960 s), projection slides of electron micrographs for talks or teaching were generally prepared as $3-1 / 2^{\prime \prime} \times 4$ "lantern slides, which were shown using large lantern-slide projectors. It was felt by professional electron microscopists that the detail, tones, and general image quality of electron micrographs could be adequately portrayed only in this larger format.

However, the large lantern slides were very cumbersome, and most professionals began switching to 2 " X 2 " slides in the early 1970 s. Some of us during that period put in a great deal of time trying to work out a procedure for printing EM negatives directly on $2^{\prime \prime} \times 2^{\prime \prime}$ glass Kodak Projector Slide Plates, following the general approach by which we had previously made the $3-1 / 4$ " $\times 4$ " lantern slides. We hoped to achieve an esthetic quality in the more convenient $2^{\prime \prime} \times 2$ " format that would approach what had been possible previously in the larger format. The following procedure developed as a result of those efforts. Making projection slides in this manner is considerably more work than the current practice of photographing EM prints on $35 \mathrm{~mm}$ film, which is certainly adequate for routine work. But if you compare the results of the two methods, you will usually see a considerable difference, and the extra work may seem worth the trouble for electron micrographs of particular quality

Kodak Projector Slide Plates, $2^{\prime \prime} \times 2^{\prime \prime}$ (51 mm $\times 51 \mathrm{~mm}$ ), are glass plates with photographic emulsion on one side. They come in two contrast grades. Contrast (cat. \#140 6875) and Medium (cat. \#140 6867). The description below is based on the Durst EM-45 enlarger in the Cell Biology Laboratories (CBL) of the Department of Anatomy and Cell Biology here at the University of Michigan. The Durst should be configured as follows (the arrangement is basically similar for $3-1 / 4^{\prime \prime} \times 4^{\prime \prime}$ EM negatives, $4^{\prime \prime} \times 5$ " negatives or $35 \mathrm{~mm}$ negatives

\section{Setup}

1) Carefully remove the point filament bulb from the Durst (without touching the glass with bare fingers), and insert large white tungsten bulb.

2) Connect. Durst-Variac (Powerstat)-Gralab timer-wall plug. Then turn on power (wall switch)

3) Set Variac at about 60 (find best position empirically).

4) Condensers: 240RT over 200T Check diagram above cover for proper orientation.

5) Appropriate negative carrier (usually $3-1 / 4^{\prime \prime} \times 4^{\prime \prime}$ )

6) $150 \mathrm{~mm}$ lens (Rodenstock Rodagon, 1:5.6/150).

7) Omega grain focuser (Omega catalog $\# 468-009$ )

8) Basebox to support $2^{\prime \prime} \times 2^{\prime \prime}$ photographic plate.

9) Two pans on sink drainboard: (1) Kodak D-19 (full strength), (2) Kodak rapid tix

10) Fill the sink with running water by fitting the metal tube in the exit drain Don t iet it I un over! Make sure water temperature control on the wall is set at about 680 degrees $F$ (previously run nearby water faucet until water becomes hot)

\section{Procedure}

1) Turn on the enlarger and make sure the white bulb is approximately centered, judged by observing its position (bulb chamber open) with relation to the optical path of the enlarger. Turn off the light

2) Place the 3-1/4" $\times 4^{\prime \prime}$ EM negative in the carrier, emulsion side down, and remove any dust with a blower can. Place the carrier in the enlarger 3) Turn on the light and obtain the desired image on a test $2^{\prime \prime} \times 2^{\prime \prime}$ Kodak plate on the basebox. The lens diaphragm should be wide open, for adequate light and narrow dept of focus). Change magnification by means of bellows (shorter bellows for higher magnification)

4) Hocus carefully (spherical knob) on the grain of the negative, using the Omega grain focuser Focus with the lens diaphragm open and then close down to abuut f11-f16 Set light level fairly low, to give an exposure of (Continued on last page)

\section{～The Link ISIS factor}

The value of time is known in all laboratories. The sheer speed of Link ISIS gives you the extra time you need. Extra time to analyze more samples. Extra time to spend on the interpretation of your results rather than just acquiring data.

From acquisition to report, Link ISIS is the fastest EDX system around.

* $\quad 10$ element quant in under 1 second

* $\quad 32$ maps in 4 seconds

* Beamsearch - the fastest possible way to find an area of interest $30,000 \mathrm{cps}$ acquisition rate

Accurate auto ID of all elements in under 1 second

Super fast spectrum movement

Compare for yourself, no other system can match the performance of Link ISIS. Fast data acquisition, fast processing, fast report generation, fast applications support and fast becoming the only EDX system worth considering.

Link ISIS gives you the extra time you need - so perhaps it's time you took a closer look!

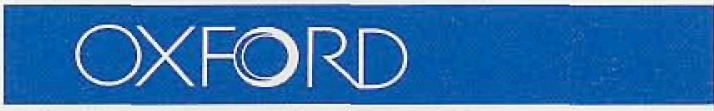

Oxford Instruments Microanalysis Group

130A Baker Ave. Ext. Concorde, MA 01742-2204

Tel: 5083699933 Fax: 5083698287
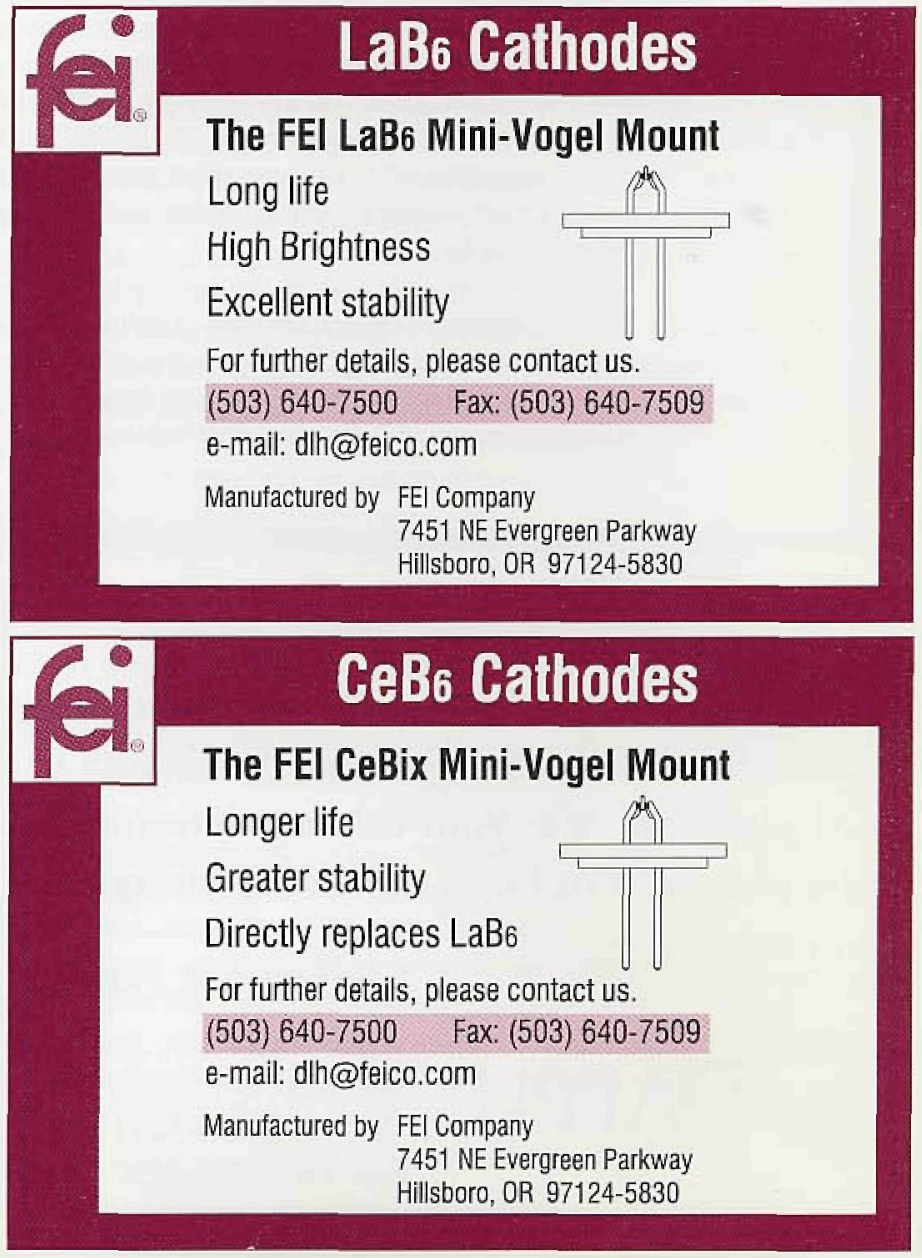


\section{Automatic Particle Analysis - Continued}

analytical substrate and its interaction with any dispersant. The high polish required of the analytical substrate (needed so that your dumb computer won't find and size substrate defects) sometimes leads to unexpected wetting behavior.

Establishing the analytical parameters is the part of the automated analysis that will seem most familiar to a microscopist. As a general rule, what looks good to you will look good to the computer, but keep in mind that the computer will recognize particles by contrast, so that any imaging or illumination mode should maximize the difference in brightness between the particles and the background without flaring the particles, which will increase their apparent size. In light microscopy, darkfield illumination is a good choice. In the SEM, backscattered electrons are often used if a suitable substrate with an atomic number much different from the particles can be found--we usually use polished carbon or aluminum, occasionally beryllium. In some cases you may want to acquire an image in which the partivcles are dark and the substrate is bright, for instance clay on gold. What matters is the difference in brightness, not the direction.

The digital image that you acquire is actually a stream of digits defining the location of each picture element (pixel) in a two dimensional grid and the intensity of the imaging signal, for example, backscattered electron current, at that pixel location. (The code that tells your computer how to assemble these numbers into an image is the "image format." PICT and TIFF are the two most common, but many others are used.) When these numbers are appropriately assembled and represented on your computer screen, you see an image, but the computer sees a two dimensional matrix, with entries representing signal intensity. Once you have this digital image, the possibilities for manipulating it are nearly unlimited. The most common adjustments are to the image brightness and contrast, which need not be linear, and can imitate any function. This step is entertaining and creative, but if you have to take it, you probably didn't prepare and setup your analysis properly

Usually the computer recognizes a particle as a set of contiguous pixels with a signal intensity above a background level which you establish. Some programs require that you first convert the image to a binary, which sets all pixels above the background to ' 1 ' and all those below to ' 0 ', others allow you to define "particles" as features within a set range of the signal intensity. Most programs work only with grayscale images with a full range of 256 , but a few are able to handle color images, which contain much more information One of the major advantages of advanced microscopy based automated particle analysis comes into play for samples that consist of a mixture of particle types. If you can differentiate different types from one another by color or brightness, then the data for each type can be compiled separately, allowing for example, the simultaneous but separate determination of iron oxide and carbon black particle morphologies in copier toner, an impossible feat for non-microscopical techniques.

Once the particles are recognized by the computer as distinct features, they can be measured. A great number of possible measurements exist, and the ones you choose will depend on why the analysis is being done in the first place. The most common measurements are maximum diameter, minimum diameter and average diameter. Besides these mundane parameters, things like particle perimeter, Feret diameters, convex area or circularity may be requested. The algorithm may also record the position of the particle. For systems integrated into the electron microscope, this ability allows EDS analyses of each particle to be done and the resulting elemental data to be stored.

All of the data you so collect is worthless if it cannot be communicated. It may be enough for you to hand a disk with raw data to the person who needs it, but its not very fulfilling. Most of the challenge involved in automated particle analysis (once you've figured out how to set it up) comes in trying to understand the data, and there is a lot of it. measurement of six morphological parameters and ten element $x$-ray intensities on two thousand particles is not an unusual case, giving thirty two thousand data points. Plotting by hand is not going to work. What does work is more automation, either through commercial spreadsheet or statistical programs which will accept the data in tabular form, or similar functions provided as part of the automation software. In either case, the common results are tables of summary descriptive statistics (mean, median, confidence interval, skewness, etc.) and histograms of the distributions of morphological features. When particle types can be differentiated on some basis, then all of these statistics and graphs are usually given separately for each particle type. This capability alone is worth the cost of the system, for there is no other way to measure the characteristics of different particle types in a mixture.

Automated particle analysis brings a new dimension of capability to the microscopist--ease of quantitation. Now you can report data for hundreds or thousands of individual particles and make statistically significant inferences about particulate samples without the tedium of hours spent accumulating the data. When you use these systems properly, the computer does what it does best--boring, repetitive chores requiring great precision, and you are freed to think about what it all means.

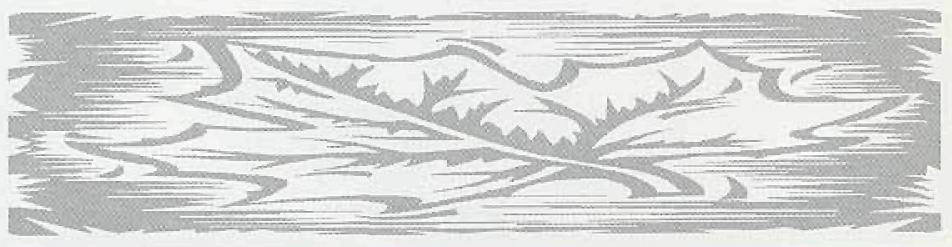
2" X 2" Projection Slides - Continued

approximately 10-20 seconds (enough time to dodge or burn in, if necessary). 5) With the room dark, except for sodium vapor lamp (which should be set with cover at darkest level), place an unexposed $2^{\prime \prime} \times 2^{\prime \prime}$ plate into position on the basebox. Expose a sufficient length of time to insure a development time of about 2 minutes. The exposure time can be determined by carrying out an exposure series on a single plate (upgraded exposures across the plate; perhaps start with $40,30,20,10 \mathrm{sec}$.). When the optimal time has been determined, expose a plate. If the electron micrograph is important, then several alternatives can be made with slight variation in exposure, with dodging (if necessary) or other effects.

6) Develop in a pan of Kodak D-19 or D-11 developer (undiluted) for 2 minutes, with continuous agitation for the first minute and no agitation thereafter. D-19 will give good contrast; use D-11 if still more contrast is needed. If you want less contrast, ty Dektol (1:2 in water).

7) Rinse plates briefly in running water, and then fix about 3-5 minutes in a pan of rapid fix (or 10 minutes in ordinary Kodak fixer).

8) Put the plates in a plastic plate holder submerged in the sink, and allow them to wash for about 30 minutes in running water. (If you prefer you can use hypo-clearing agent for 2 minutes after fixation, followed by a 10 minute wash). Dip for about 10 seconds in Photoflo (suitably diluted, according to instructions), then place the plastic plate holder in a drying oven (as dustfree as possible) for drying.

9) Place the dried plates on a light box or viewer, and select the optimal slide for each electron micrograph. Be careful not to scratch the emulsions while handling them. If you find them difficult to judge, you may want to place a temporary coverslip on the emulsion side, put them in a projector and then project them on a screen to decide.

10) Bind the final projection slides as follows: Frame the optimal field by masking with silver tape (in addition to framing the field, the masking also acts as a spacer to keep the coverglass from touching the emulsion, which might produce Newton rings). With a blower can, remove any dust from the emulsion and from one side of a clean coverglass. Put the coverglass on the emulsion side of the plate, and slip both the plate and coverglass into an Emde aluminum $2^{\prime \prime} \times 2^{n}$ slide binder, making sure that the emulsion side of the plate faces the complete side of the binder. Bend over the free edge of the binder being careful to support the adjacent side of the binder so it does not bulge out.

11) Information about the slide can be witten on the aluminum binder with a ball-point pen (which writes by engraving the soft metal surface). 